\title{
Quantum-Induced Symmetry-Breaking in the Deuterated Dihydroanthracenyl Radical
}

Olha Krechkivska, Callan Wilcox, Klaas Nauta, Scott Kable, Timothy Schmidt

Submitted date: 14/05/2019 - Posted date: 15/05/2019

Licence: CC BY-NC-ND 4.0

Citation information: Krechkivska, Olha; Wilcox, Callan; Nauta, Klaas; Kable, Scott; Schmidt, Timothy (2019): Quantum-Induced Symmetry-Breaking in the Deuterated Dihydroanthracenyl Radical. ChemRxiv. Preprint.

The hydrogen-atom adduct with anthracene, 9-dihydroanthracenyl radical $\left(\mathrm{C}_{14} \mathrm{H}_{11}\right)$, and its deuterated analogue, have been identified by laser spectroscopy coupled to time-of-flight mass spectrometry, supported by time-dependent density functional theory calculations. The electronic spectrum of 9-dihydroanthracenyl radical exhibits an origin band at $19115 \mathrm{~cm}^{-1}$ and its ionization energy was determined to be $6.346(1) \mathrm{eV}$. The spectra reveal a low-frequency vibrational progression corresponding to a mode described by a butterfly-inversion. In the deuterated analogue, a zero-point-energy imbalance along this coordinate is found to lead to a doubling of the observed spectral lines in the progression. This is attributed to quantum-induced symmetry breaking as previously observed in isotopologues of $\mathrm{CH}_{5}{ }^{+}$.

File list (3)

AnthraceneH.pdf (4.47 MiB)

view on ChemRxiv • download file

SI.pdf (524.51 KiB) view on ChemRxiv • download file view on ChemRxiv • download file 


\title{
Quantum-induced symmetry breaking in the deuterated dihydroanthracenyl radical
}

\author{
Olha Krechkivska, ${ }^{1}$ Callan M. Wilcox, ${ }^{1}$ Klaas Nauta, ${ }^{1}$ Scott H. Kable ${ }^{1}$ \\ and Timothy W. Schmidt ${ }^{1,2 *}$
}

1. School of Chemistry, UNSW Sydney, NSW 2052, Australia

2. ARC Centre of Excellence in Exciton Science, Australia

*To whom correspondence should be addressed; E-mail: timothy.schmidt@unsw.edu.au.

\begin{abstract}
The hydrogen-atom adduct with anthracene, 9-dihydroanthracenyl radical $\left(\mathrm{C}_{14} \mathrm{H}_{11}\right)$, and its deuterated analogue, have been identified by laser spectroscopy coupled to time-offlight mass spectrometry, supported by time-dependent density functional theory calculations. The electronic spectrum of 9-dihydroanthracenyl radical exhibits an origin band at $19115 \mathrm{~cm}^{-1}$ and its ionization energy was determined to be $6.346(1) \mathrm{eV}$. The spectra reveal a low-frequency vibrational progression corresponding to a mode described by a butterflyinversion. In the deuterated analogue, a zero-point-energy imbalance along this coordinate is found to lead to a doubling of the observed spectral lines in the progression. This is attributed to quantum-induced symmetry breaking as previously observed in isotopologues of $\mathrm{CH}_{5}^{+}$.
\end{abstract}




\section{Introduction}

Polcyclic aromatic hydrocarbons (PAHs) are of broad general interest. They are considered by some to be nano-graphenes, $[1,2]$ are by-products of combustion, $[3,4]$ and pose significant environmental health risks.[5] There is also substantial evidence for their widespread abundance in astrophysical environments. While the first definitive identification of an interstellar aromatic species was reported only in 2018,[6] PAHs have long been held responsible for several infrared emission features observed in post asymptotic giant branch stars, young stellar objects and galactic nuclei.[7]

Given the abundance of hydrogen atoms in the interstellar medium (ISM), it is of interest to study the interaction of hydrogen with prototypical PAHs. Indeed, PAHs are thought to act as catalysts for the formation of molecular hydrogen in Space.[8-11] Reversible addition of $\mathrm{H}$ to PAHs provides a pathway to deuterium enrichment in the ISM.[12] Sandford and coworkers concluded that PAHs can be enriched with deuterium by ion-molecule reactions in dense molecular clouds, and PAHs represent the largest reservoir for D enrichment of all ionmolecule reactions.

The radicals formed by $\mathrm{H}$-addition to PAHs are resonance-stabilized.[13] This stabilization of radicals allows their concentrations to build up in reactive and harsh environments, such as in combustion $[4,14,15]$ and are postulated to occur in the interstellar medium.[16] The association of hydrogen with benzene breaks aromaticity and forms the cyclohexadienyl radical $\left(\mathrm{C}_{6} \mathrm{H}_{7}\right),[17]$ an important class of intermediate involved in many processes, such as the atmospheric oxidation of benzene.[18, 19] Addition of hydrogen to naphthalene can occur at one of two unique locations on the ring, forming the 1- and 2-dihydronaphthyl radicals $\left(\mathrm{C}_{10} \mathrm{H}_{9}\right)$. [2022] The spectroscopy and thermochemistry of the 1- and 2-dihydronaphthyl radicals were ex-

plored by Zwier and co-workers to aid in the photochemical models of PAH formation in the 
atmosphere of Titan.[23]

There has been little attention given to the $\mathrm{H}$-adduct with anthracene, the dihydroanthracenyl radical $\left(\mathrm{C}_{14} \mathrm{H}_{11}, \mathrm{H}-\mathrm{An}\right)$. Maier and co-workers observed a dihydroanthracenyl radical in a neon matrix.[24] They observed a broad absorption feature in the ultraviolet with an origin at $326 \mathrm{~nm}$. The location of the additional hydrogen on the anthracene scaffold was not determined, with the authors reporting that the spectrum may relate to $\mathrm{H}$-addition at the 1 and or 9-positions.

In this work, we identify the 9-dihydroanthracenyl radical by resonance-enhanced ionization spectroscopy supported by density functional theory calculations. As in previous work,[22] we also added a deuterium atom and find that a zero-point-energy imbalance along an out-of-plane bending coordinate leads to quantum-induced symmetry-breaking, as previously observed in $\mathrm{CH}_{5}^{+},[25]$ with striking spectroscopic consequences.

\section{Experimental}

The experimental apparatus and methods have been described previously.[22, 26] Briefly, a sample of anthracene was heated in the range $75-100^{\circ} \mathrm{C}$ before a pulsed discharge nozzle, which was held $\sim 10^{\circ} \mathrm{C}$ higher to prevent condensation of the sample. A gas mixture of argon and (heavy) water was passed through the sample container, collecting the vapour of anthracene. The (heavy) water was heated externally to $50^{\circ} \mathrm{C}$.

A discharge of $2.1 \mathrm{kV}$ was struck for $130 \mu$ s during the gas expansion, with current limited by a $20 \mathrm{k} \Omega$ ballast resistor. Following the discharge, the mixture was supersonically expanded into a differentially-pumped vacuum chamber and the coldest part of the expansion was admitted through a $2 \mathrm{~mm}$ skimmer. To search for the $\mathrm{H}$ and $\mathrm{D}$-adducts of anthracene, a resonant 2-color 2-photon ionization (R2C2PI) scheme was employed. An ionisation source of $\sim 290 \mathrm{~nm}$ was used. The $m / z 179$ and 180 signals, (corresponding to H-An and D-An), were recorded as a function of excitation wavelength. 
The ionization energy of the radicals was measured by fixing the excitation laser to the origin (or a low-lying vibrational band) of the radical's electronic excitation, and scanning the ionization laser. The excited-state lifetime of the H-adduct was obtained by fixing the timing of the ionization pulse, and scanning the timing of the preceding excitation laser pulse. The trace was fit to an exponential decay.

Hole-burning experiments were conducted on the deuterium adduct, to confirm the commonality of the spectral carrier of individual excitation bands. With the excitation laser fixed on a particular band, a hole-burning laser was introduced about $500 \mathrm{~ns}$ prior, to remove population from the ground state and thus deplete the resonant ionization signal. The wavelength of the hole-burning laser was scanned to reveal the excitation spectrum of the species probed by the R2C2PI experiment.
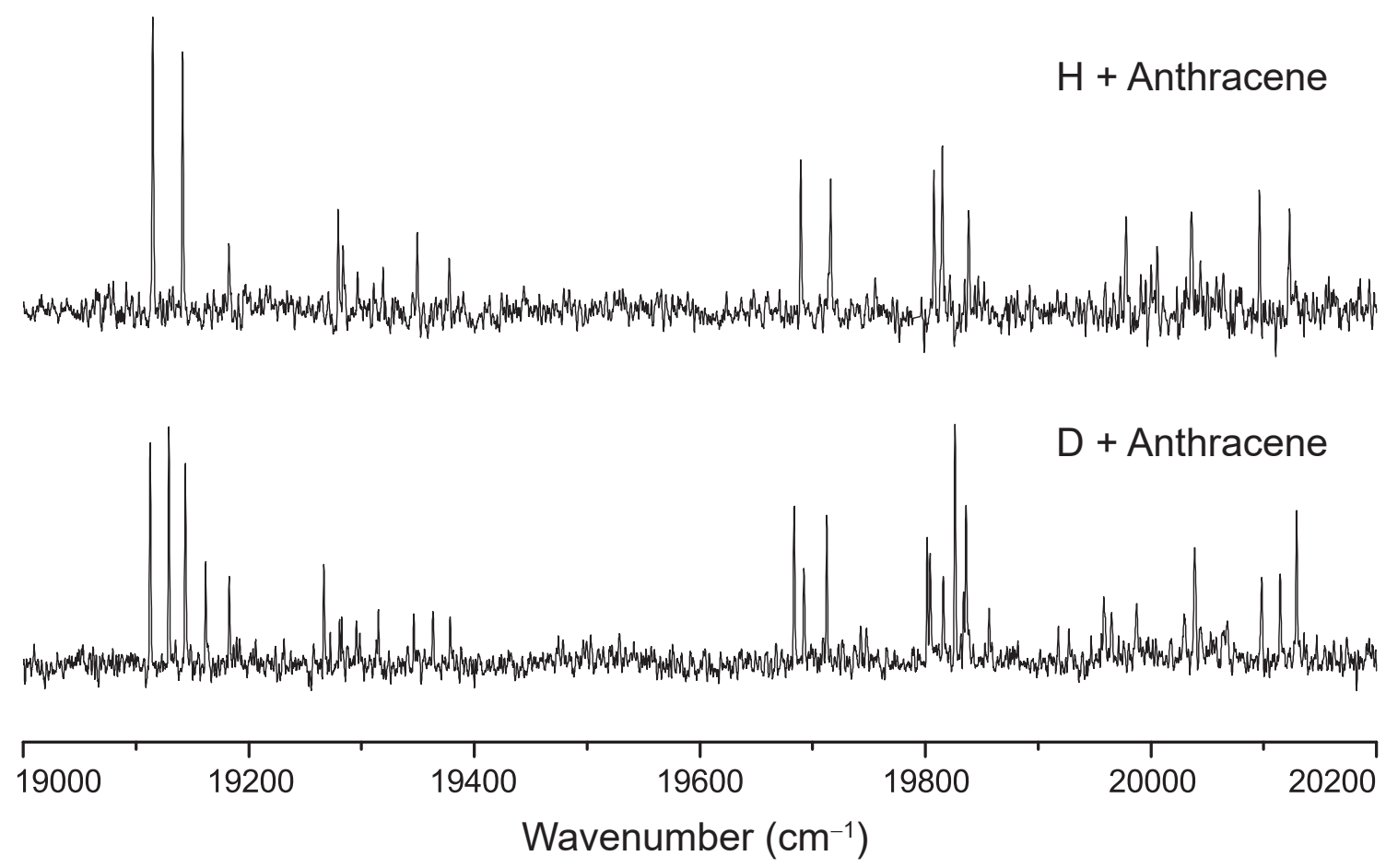

Figure 1: R2C2PI spectra of a dihydroanthracenyl radical $\left(\mathrm{H}-\mathrm{An}, \mathrm{C}_{14} \mathrm{H}_{11}\right)$ and the deuterated analogue (D-An, $\left.\mathrm{C}_{14} \mathrm{H}_{10} \mathrm{D}\right)$. 


\section{Results}

R2C2PI spectra were recorded for $m / z 179$ and 180 , corresponding to adducts of anthracene with a hydrogen or deuterium atom respectively (H-An, $\mathrm{C}_{14} \mathrm{H}_{11}$ and D-An, $\mathrm{C}_{14} \mathrm{H}_{10} \mathrm{D}$ ). The spectra are plotted in Figure 1. The spectra exhibit rich vibrational structure with multiple peaks near the origin for both species.

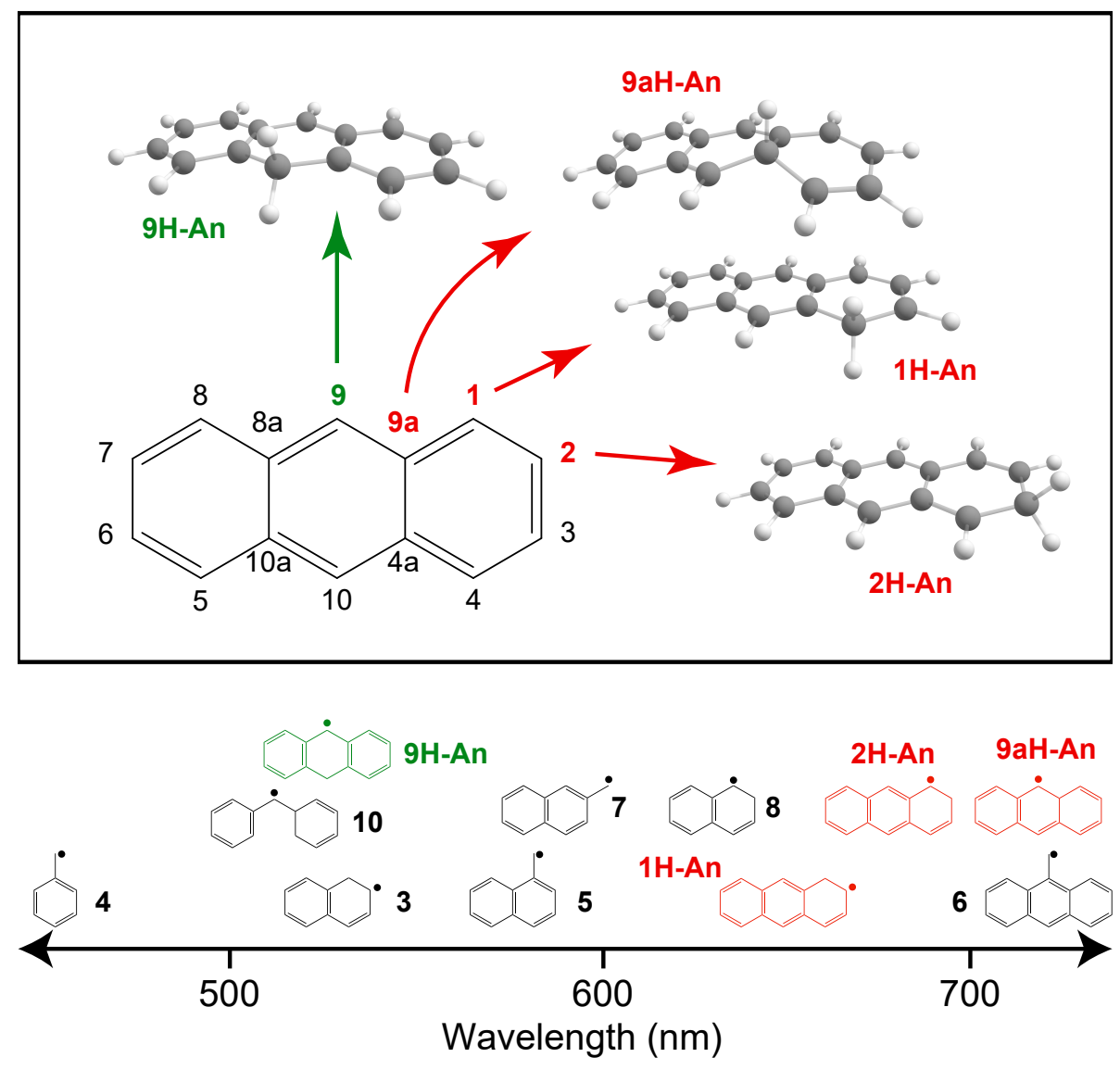

Figure 2: Numbering convention for anthracene derivatives with unique substitution locations shown in bold, pointing to structures of $\mathrm{H}$-An radicals. See text for a discussion of previously observed radicals with chromophores similar to those of the four dihydroanthracenyl radical isomers. 


\subsection{Identification of the 9-dihydroanthracenyl radical}

There are four symmetry-distinct sites at which a hydrogen atom may attach to anthracene: At the 1-, 2-, 9- and 9a-positions (Figure 2). The observed species at $m / z 179$ presents a putative origin band at $523 \mathrm{~nm}\left(19115 \mathrm{~cm}^{-1}\right)$ and we use this information to propose the identity by comparison to known spectra.

The chromophore presented by the 1-dihydroanthracenyl radical (1H-An) could be considered a benzannulated 1-dihydronaphthyl radical ( 3 in Figure 2). This radical, which has a phenylallyl-type chromophore, was observed by Zwier and co-workers to absorb at $528 \mathrm{~nm}$.[23] However, benzannulation shifts the absorption to longer wavelength.[13] For instance, benzyl radical (4) absorbs at $454 \mathrm{~nm}, 1$-naphthylmethyl radical (5) absorbs at $580 \mathrm{~nm}$,[27] and 9anthracenylmethyl (6) absorbs at $727 \mathrm{~nm}$.[28] As such, we would expect 1-dihydroanthracenyl radical to absorb some $\sim 130 \mathrm{~nm}$ to longer wavelength than the observed 1-hydronaphthyl origin, i.e. near $658 \mathrm{~nm}$.

The 2-dihydroanthracenyl radical (2H-An) chromophore is similar to the 2-naphthylmethyl radical (7) substituted with an ortho vinyl group. Vinyl substitution tends to shift absorptions to lower wavenumber (longer wavelength) by about $2500 \mathrm{~cm}^{-1}$.[13] A vinyl-substituted 2naphthylmethyl radical chromophore would therefore be expected to absorb redward of $584 \mathrm{~nm}$.[27]

The 2-dihydroanthracenyl radical may also be considered a benzannulated 2-dihydronaphthyl radical (8). The latter radical is thought to absorb near $636 \mathrm{~nm}$,[29] and a benzannulated version would almost certainly absorb redward of that figure.

The 9a-dihydroanthracenyl radical (9aH-An) is not expected to be particularly stable on account of the induced ring strain, and the equivalent $\mathrm{H}$ adduct with naphthalene has not been observed.[20] Such a radical would exhibit a nine-membered $\pi$-chromophore with benzannulation. The pentadienyl chromophore absorbs near $514 \mathrm{~nm}$,[30] which is $\sim 106 \mathrm{~nm}$ redward of the three-membered allyl chromophore.[31] The nine-membered $\pi$-chromophore on its own would 
therefore be expected to absorb at some $726 \mathrm{~nm}$, far to the red of the observed spectrum.

The 9-hydroanthracenyl radical (9H-An) exhibits a very similar chromophore to the diphenylmethyl ( $\alpha$-phenylbenzyl) radical (10), which has been studied previously.[32] Shibuya and coworkers assigned the origin of this radical to a feature observed at $516 \mathrm{~nm}\left(19370 \mathrm{~cm}^{-1}\right)$, with extensive Franck-Condon activity observed in torsional modes. The close proximity to the absorption position of the presently observed spectrum strongly suggests the identity to be 9hydroanthracenyl radical.

Photo-ionization spectra were obtained for each of the peaks in the origin regions of the H-An and D-An spectra. The results are displayed in Figure 3. The top two spectra result from exciting the origin and the first successive transitions in the $\mathrm{H}$-adduct spectrum, and are labelled $h_{a}$ and $h_{b}$. The point of intersection between the baseline and the ionization onset is more clearly defined for $h_{a}$, and so we have used this spectrum to determine the ionization energy. This range encompasses the value obtained by ionizing through $\mathrm{h}_{\mathrm{b}}$ and can be considered consistent. In previous experiments we determined that for similar sized radicals, the finite electric field in the extraction region was responsible for a depression of the observed IE of $7 \mathrm{meV}$. As such, our recommended value is $6.3462(10) \mathrm{eV} .[33]$

Time-dependent density functional theory calculations were performed to support the above identification of the spectral carrier. (TD)-B3LYP calculations were carried out using the Gaussian 16 suite of programs[34] and the 6-311+G(d,p) basis set. The adiabatic (and ZPE corrected) ionization energies of the $1 \mathrm{H}-\mathrm{An}, 2 \mathrm{H}-\mathrm{An}, 9 \mathrm{aH}-\mathrm{An}$ and $9 \mathrm{H}-\mathrm{An}$ isomers were also calculated at the B3LYP/6-311+G(d,p) level of theory, and the results are reported in Table 1. All four isomers are predicted to have similar ionization energies and, characteristic of the B3LYP/6$311+\mathrm{G}(\mathrm{d}, \mathrm{p})$ level of theory, the values are lower than the observed value.[33, 35]

For a range of resonance-stabilized radicals, Troy reported that the B3LYP/6-311+G(d,p) adiabatic ionization energies underestimated experiment by a deviation of 0.14(7) eV.[35] By 

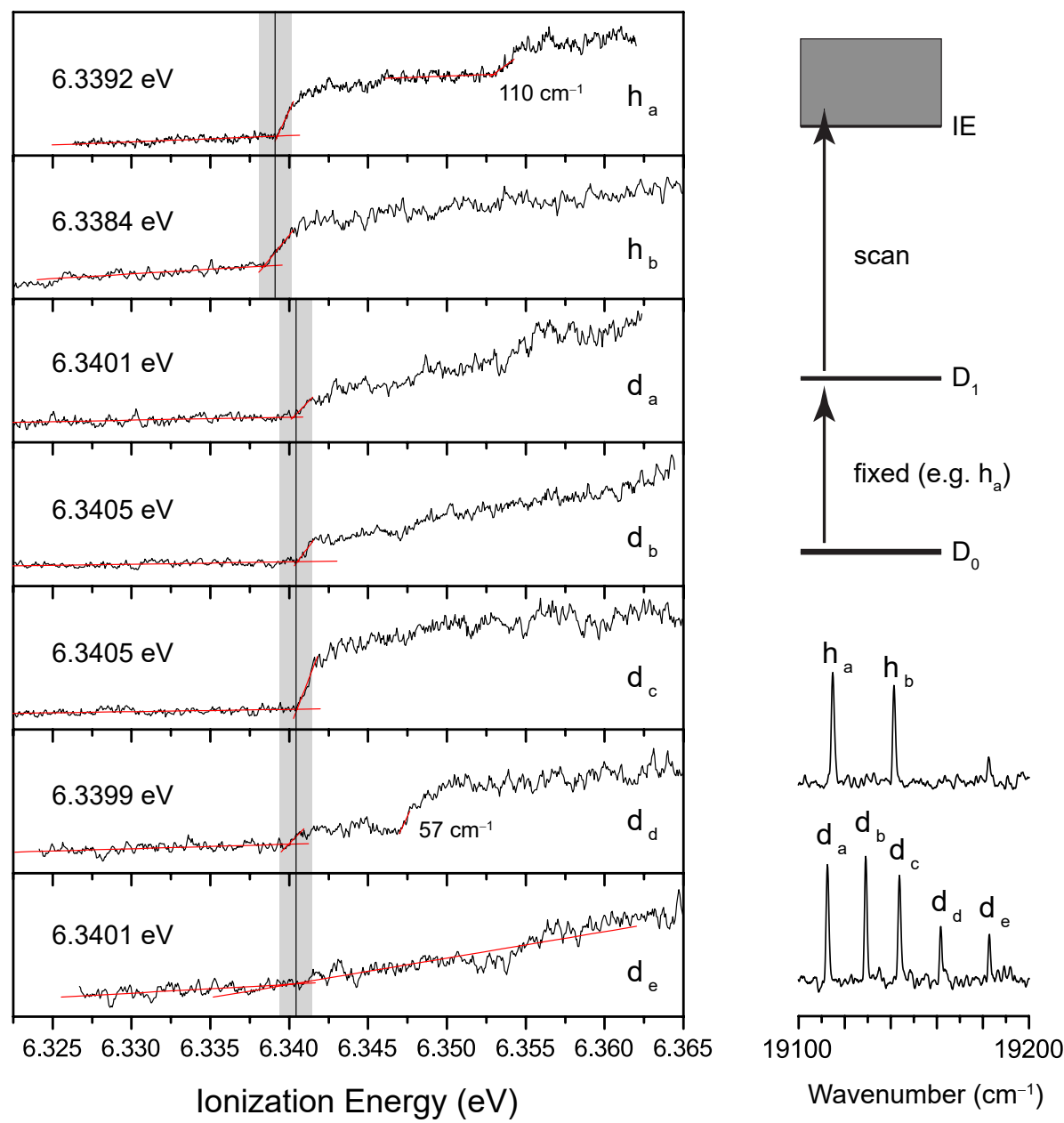

Wavenumber $\left(\mathrm{cm}^{-1}\right)$

Figure 3: Photo-ionization spectra of the H-An $\left(h_{a}\right.$ and $\left.h_{b}\right)$ and D-An radicals $\left(d_{a}\right.$ to $\left.d_{e}\right)$, with the first excitation through the band indicated. Top right is a schematic of the ionization scheme. The reported ionization energy in the text and Table 1 is corrected by $+0.007 \mathrm{eV}$ to account for the finite electric field in the extraction region of the spectrometer.[33]

adding the $0.14(7) \mathrm{eV}$ to the calculated adiabatic energies, we obtain our best calculated estimates for the ionization energies of the various isomers. All but 9aH-An radical have calculated IEs consistent with the experimentally observed value of $6.346 \mathrm{eV}$. As such we cannot use the ionization energy alone as a way to discriminate between the isomers. However, they are consistent with the carrier being assigned to an H-An species (other than 9aH-An).

The calculated relative energies of $1 \mathrm{H}-\mathrm{An}, 2 \mathrm{H}-\mathrm{An}, 9 \mathrm{aH}-\mathrm{An}$ and $9 \mathrm{H}-\mathrm{An}$ are given in Ta- 
ble 1. The 9H-An isomer is the most energetically favourable to form, by $27.7,45.6$ and $136.8 \mathrm{~kJ} \mathrm{~mol}^{-1}$ compared to addition at the 1-, 2- and 9a- positions respectively. While an electric discharge is capable of generating higher energy isomers,[26] the low energy calculated for $9 \mathrm{H}-$ An supports its assignment as the observed carrier.

The adiabatic $\mathrm{D}_{1} \leftarrow \mathrm{D}_{0}$ excitation wavelengths for the $1 \mathrm{H}-\mathrm{An}, 2 \mathrm{H}-\mathrm{An}$ and $9 \mathrm{H}-\mathrm{An}$ isomers were also calculated at the TD-B3LYP/6-311+G(d,p) level, including their respective zero-point energy corrections (Table 1 ). The TD-B3LYP geometry optimization in the $D_{1}$ state of the $9 \mathrm{aH}-$ An isomer failed to converge. The calculated absorption wavelengths are consistent with the assertions in Figure 2, which were based on comparing chromophores.

The observed H-An electronic origin transition is ascribed to the first peak in the R2C2PI spectrum, at $19115 \mathrm{~cm}^{-1}$. No bands were observed to the red of this transition. The calculated $\mathrm{D}_{1} \leftarrow \mathrm{D}_{0}$ transition for the $9 \mathrm{H}-\mathrm{An}$ isomer, at $20101 \mathrm{~cm}^{-1}$, is to slightly higher energy than the observed value, with a discrepancy of $986 \mathrm{~cm}^{-1}$. The $1 \mathrm{H}-\mathrm{An}$ and $2 \mathrm{H}-\mathrm{An}$ isomers are calculated to absorb some $2462 \mathrm{~cm}^{-1}$ and $3591 \mathrm{~cm}^{-1}$ lower in energy, respectively, than $9 \mathrm{H}-\mathrm{An}$. On the basis of the observed origin band position and the relative isomer energies, we assign the observed spectrum to the 9-dihydroanthacenyl radical, 9H-An.

\subsection{Spectral Hole-burning}

With the identity of the $9 \mathrm{H}-\mathrm{An}$ radical secure, we note a curious difference between the deuterium and hydrogen-adducts in Figure 1. The higher resolution spectra of the origin regions (see Figure 3) reveal a halving of the observed low frequency mode upon deuteration - something which is not possible due to the mass difference alone. To check that all bands originate in a single isotopologue, and not from scrambling of the D-position, we performed hole-burning experiments.

In order to perform a hole-burning experiment and remove population from the $v=0$ level, 
Table 1: Calculated (TD)-B3LYP/6-311G+(d,p) Energies, Excitation Wavelengths and Ionization Energies of $\mathrm{C}_{14} \mathrm{H}_{11}$ Isomers

\begin{tabular}{cccc}
\hline \hline Isomer & $\begin{array}{c}\text { Rel. Energy }^{a} \\
\left(\mathrm{~kJ} \mathrm{~mol}^{-1}\right)\end{array}$ & $\begin{array}{c}\text { Excitation } \\
(\mathrm{nm})\end{array}$ & $\begin{array}{c}\mathrm{IE}^{a, b} \\
(\mathrm{eV})\end{array}$ \\
\hline $1 \mathrm{H}-\mathrm{An}$ & 27.7 & 600 & $6.38(7)$ \\
$2 \mathrm{H}-\mathrm{An}$ & 45.6 & 644 & $6.34(7)$ \\
9H-An & 0 & 497 & $6.29(7)$ \\
9aH-An & 136.8 & unconverged & $6.16(7)$ \\
\hline Recommended & & 523 & $6.3462(10)^{c}$
\end{tabular}

a ZPE corrected

$b$ Adiabatic, corrected by $+0.14(7) \mathrm{eV}$ (see text)[35]

$c$ Corrected by $+0.007 \mathrm{eV}$ to account for the finite electric field in the extraction region of the spectrometer[33]

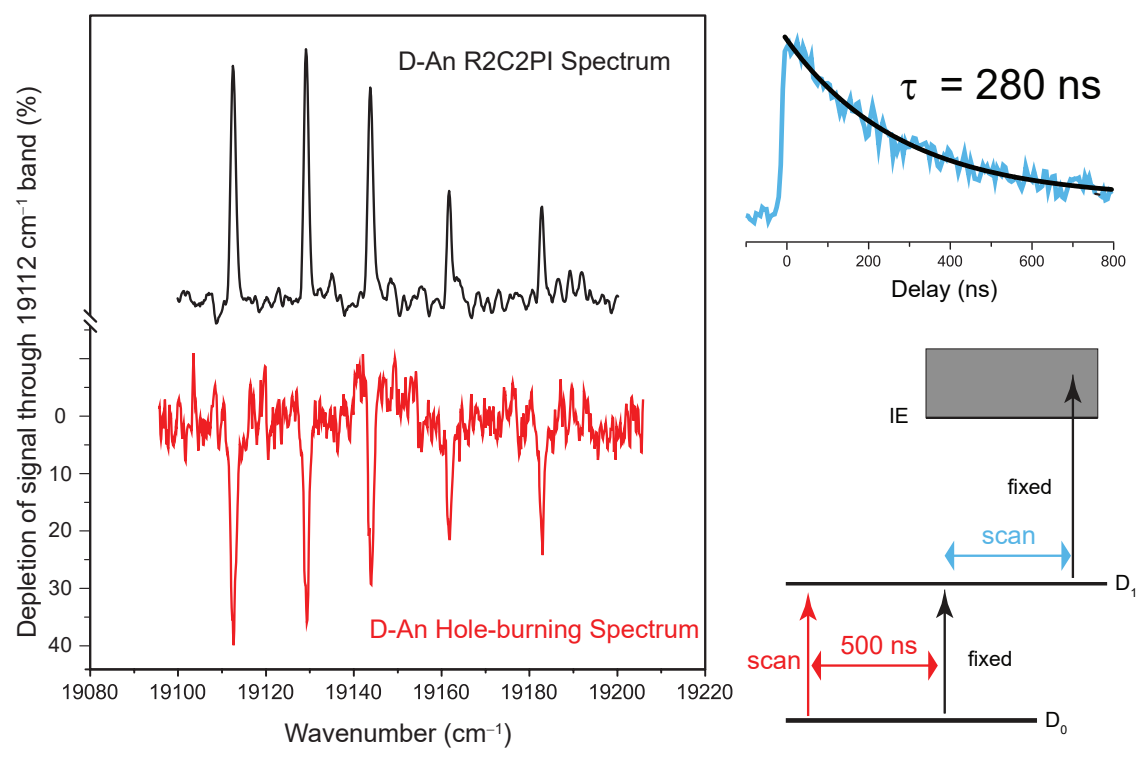

Figure 4: Hole-burning spectrum of the D-An radical probed through the origin band. As all features are present in both spectra, we can conclude that they belong to the same ground state species. At top right is the scan of the excitation-ionization delay which reveals the excited state lifetime. At bottom right is a schematic of the lifetime and hole-burning experiments.

the hole-burning laser pulse must precede the ionization scheme by a long enough delay that the excited state decays such that it is no longer readily ionized. To this end, we performed 
an experiment in which the delay between the excitation and ionization laser pulses is scanned from negative to long positive delays. The results are plotted in Figure 4, and the trace is fit to a single exponential decay with a time constant of $280 \mathrm{~ns}$. In the hole-burning experiment, the hole-burning pulse was timed $500 \mathrm{~ns}$ prior to the ionization scheme.

Figure 4 displays two spectra. At top is the R2C2PI spectrum of the D-An electronic origin region. Fixing the R2C2PI scheme to the $19112 \mathrm{~cm}^{-1}$ origin, the hole-burning laser is scanned across the spectral range. Bands that exhibit depletion belong to the same lower state (and therefore species) as the origin. There is a clear and definitive depletion of all five bands in the D-An R2C2PI spectrum and therefore all five bands belong to the same spectral carrier and lower state as the band at $19112 \mathrm{~cm}^{-1}$. This confirms that H-D exchange does not occur to an observable extent, and that there is only one isotopologue responsible for the D-An spectrum. We assign this to the 9D-An radical.

\subsection{Spectral Assignments}

The origin regions of both the $9 \mathrm{H}-\mathrm{An}$ and 9D-An radicals exhibit a progression in a lowfrequency, anharmonic mode. This progression is reprised as a motif built onto many of the vibronic features, which is particularly clear for the two pairs of $9 \mathrm{H}-\mathrm{An}$ bands near $19700 \mathrm{~cm}^{-1}$ and $20100 \mathrm{~cm}^{-1}$.

To commence a spectral assignment, we performed excited-state frequency calculations at the TD-B3LYP/6-311+G(d,p) level. A full list of calculated frequencies is provided in the Supporting Information. For ease of comparison to experiment, we scale the calculated frequencies by 0.97.[26] Both the optimized (TD)-B3LYP/6-311+G(d,p) geometries of the $D_{0}$ and $D_{1}$ states have the $>\mathrm{CH}_{2}$ group puckered slightly out of plane, reducing the point-group symmetry from $C_{2 v}$ to $C_{s}$. As such, we have labelled and arranged the calculated modes according to the Mulliken convention in this point group. The lowest-frequency calculated mode, $\nu_{37}=36 \mathrm{~cm}^{-1}$, is 

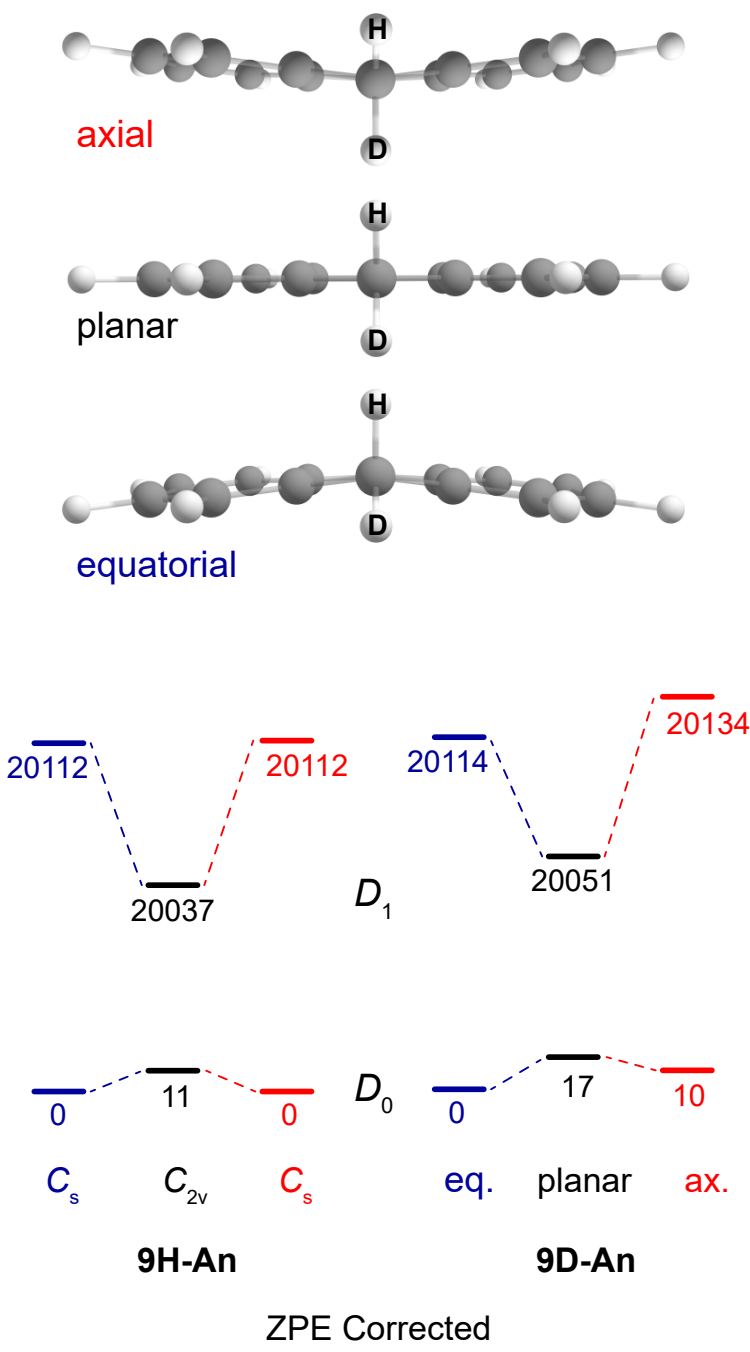

Figure 5: Top: Illustration of the butterly mode, $\nu_{37}$, highlighting the difference in the $\mathrm{D}$ position. Bottom: The ZPE-corrected (TD)-B3LYP potential energy surface of the butterly mode.

a "butterfly" motion. At the $C_{2 v}$ saddle point, this mode is of $b_{1}$ symmetry with an imaginary frequency of $43 \mathrm{icm}^{-1}$. The next lowest calculated frequency is $\nu_{69}=81 \mathrm{~cm}^{-1}$, and is $a^{\prime \prime}$ in the $C_{s}$ point group making it unlikely to have any intensity in single quanta (note that the mirror plane is perpendicular to the molecular framework). The first band encountered above the origin in the spectrum of $9 \mathrm{H}$-An lies at just $+26.6 \mathrm{~cm}^{-1}$. As such, we attribute the low frequency 
progression to $\nu_{37}$.

Comparing the 9H-An and 9D-An origin regions, it would appear that there is a doubling of the number of observed bands. A halving of the frequency is not expected, nor is it supported by calculations. We calculated the excited-state frequencies of the two $C_{s}$ 9D-An isotopomers, with the D position axial or equatorial. Both have $\nu_{37}$ at about $35 \mathrm{~cm}^{-1}$, similar to $9 \mathrm{H}-\mathrm{An}$. But, the results of hole-burning experiments rule out that the extra bands appear due to two isotopomers. We therefore conclude that there are lines missing in the spectrum of $9 \mathrm{H}-\mathrm{An}$. This implies a selection rule for the $9 \mathrm{H}-\mathrm{An}$ isomer possibly arising from a completely symmetric ground state wavefunction in $C_{2 v}$ to an excited state with a low or absent barrier to inversion. In this case, the low frequency progession in $9 \mathrm{H}-\mathrm{An}$ is assigned to $37_{0}^{2 v}$.

It is interesting at this point to draw a comparison between our spectra and the $S_{1} \leftarrow S_{0}$ spectrum of 9,10-dihydroanthracene.[36-38] This molecule is strongly bent in both ground and excited states, and shows a clear, lengthy Franck-Condon progression in the low-frequency butterfly motion. The progression in the excitation spectrum of dihydroanthracene is highly anharmonic, due to the double-well in the excited state. However, for this molecule the ground state is essentially degenerate since the barrier separating the two wells is $>600 \mathrm{~cm}^{-1}$. This means all transitions, between even-to-even and odd-to-odd states, are seen, comprising what is essentially a bent-to-bent Franck-Condon envelope with no missing bands. Contrastingly, the doubling of the number of observed transitions upon deuteration at the 9-position of 9H-An implies that we have a single symmetric ground state in $C_{2 v}$, and are missing the odd-levels in the excitation spectrum of $9 \mathrm{H}-\mathrm{An}$. The present radical should therefore be considered a $C_{2 v}$ species. For 9D-An, the asymmetry induced by the deuterium atom skews the zero-point corrected potential in both ground and excited states, returning the spectrum to the appearance of a bent-bent transition. The zero-point corrected energies at the Born-Oppenheimer minima and the $C_{2 v}$ transition states are plotted in Figure 5. It is interesting to note that the zero-point 
energies are lower for the $C_{2 v}$ geometry in the excited state than for the Born-Oppenheimer minima. The skewed nature of the 9D-An potential is clearly predicted. We return to this important point in the discussion.

Given the anharmonic nature and low frequency of $\nu_{37}$, we opted to model this coordinate empirically. Assuming that the peaks at +26.6 and $+67.7 \mathrm{~cm}^{-1}$ represent excitations of even quanta of the butterfly mode, we fit the spacings to a model quartic potential, similar to the method of Laane and Veguilla-Berdeca.[39, 40] Using the reduced mass of 3.4 amu of $\nu_{37}$ taken from the TD-B3LYP calculations, the spacings of the first three bands observed in the $9 \mathrm{H}-\mathrm{An}$ spectrum were well fit to a potential of the form $V(q)=-8.33 q^{2}+5.81 q^{4}$ (plotted in Figure 6, units of $\mathrm{cm}^{-1}$ and $\AA$ ). Two level-spacings and two parameters are likely to result in a good fit, but this potential can be tested by modelling the 9D-An spectrum.

As the 9H-An molecule proceeds along the butterfly coordinate, one $\mathrm{H}$ moves from an equatorial position to an axial position, with the other changing in the opposite sense. These geometries are equivalent for 9H-An, but they are not for 9D-An. Deuteration does not break the symmetry of the $9 \mathrm{H}-\mathrm{An}$ symmetric potential from the standpoint of the electronic energies, but the difference in the zeropoint energies of the other modes introduces an asymmetry to the double-well potential for 9D-An. The (TD)-B3LYP calculation predicts that this difference will be on the order of $10 \mathrm{~s}$ of $\mathrm{cm}^{-1}$ (Figure 5).

Terms in the potential which modulate the vibrational frequency of a particular mode $j$, while progressing along $q_{37}$ are of the form $V_{j}^{\prime}=a_{37, j} q_{37} q_{j}^{2}$, where $a_{37, j}=m_{j} \omega_{j} d \omega_{j} / d q_{37}$. Now, in a harmonic basis for all $j \neq 37$, we may integrate over these coordinates to arrive at an effective potential

$$
\begin{aligned}
V\left(q_{37}\right) & =V_{B O}\left(q_{37}\right)+q_{37} \sum_{j \neq 37} a_{37, j}\left(2 v_{j}+1\right) \frac{\hbar}{2 m_{j} \omega_{j}} \\
& =V_{B O}\left(q_{37}\right)+q_{37} \sum_{j \neq 37} \frac{\left(2 v_{j}+1\right) \hbar}{2} \frac{d \omega_{j}}{d q_{37}}
\end{aligned}
$$


where $V_{B O}$ is the Born-Oppenheimer potential and $n_{j}$ is the number of quanta in mode $j$. Where $v_{j}=0 \forall j$, this reduces to the change in the zero-point energy for all modes $j \neq 37$ along $q_{37}$.

Using the same model potential as for $9 \mathrm{H}-\mathrm{An}$, with a linear term of $+13.34 q$ to account for an offset in the zero point energies of the two wells, the level spacing in 9D-An was matched to experiment (reduced mass from (TD)-B3LYP, 3.7 amu). To model the appearance of the intensities, the ground state potential was modelled as $V(q)=(-0.99 q)-8.30 q^{2}+1.69 q^{4}$, where the linear term is only invoked for the 9D-An radical.
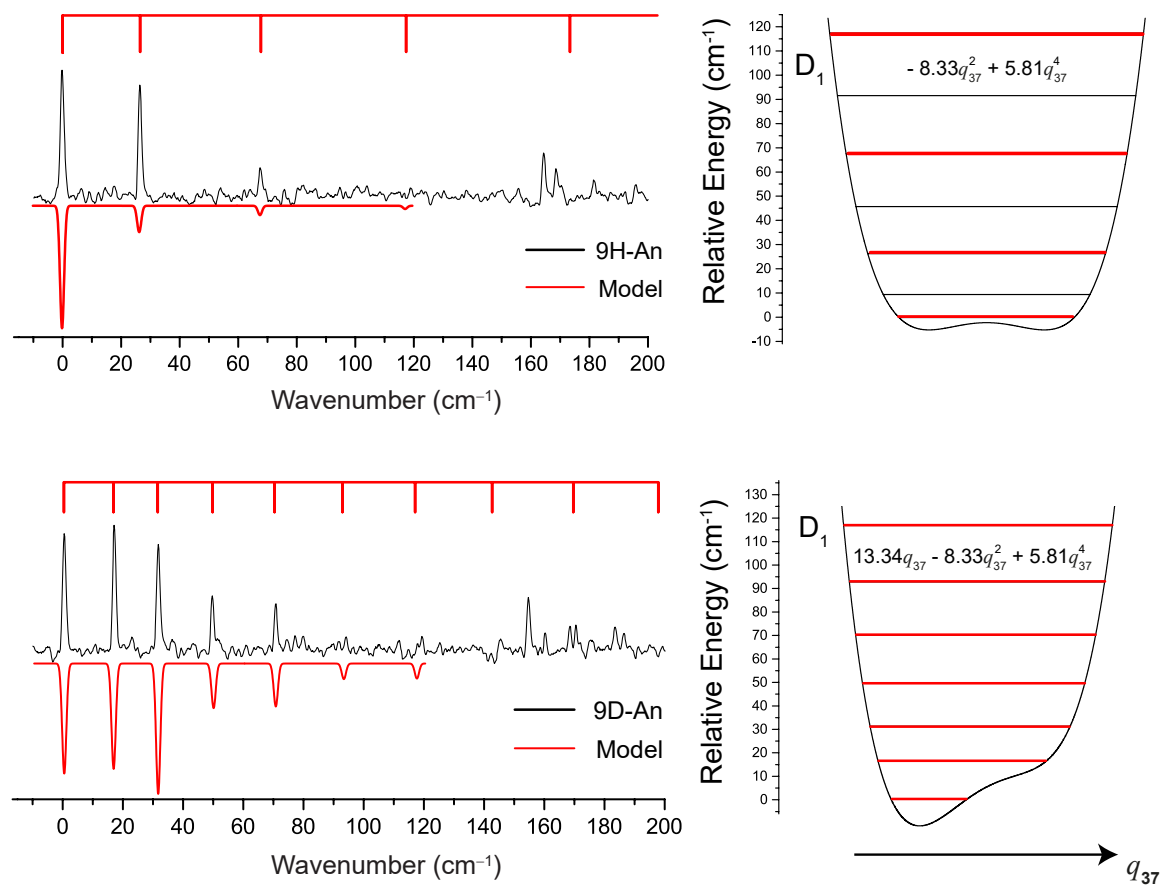

Figure 6: Model butterfly potentials and excitation spectra for the origin regions of $9 \mathrm{H}-\mathrm{An}$ and 9D-An.

With the above assigment of the origin region and the activity of $\nu_{37}$ secure, we proceeded to assign the remainder of the spectra in Figure 1 using the frequencies calculated by TD-DFT. For the bulk of the spectroscopic assignment, we refer the reader to the Supporting Information, but a short list of assignments for the $9 \mathrm{H}-\mathrm{An}$ isotopologue is given in Table 2. The full list of calculated frequencies at the Born-Oppenheimer minima is given in Tables S2-S4. 
Given the appearance of selection rules in the excitation of $\nu_{37}$, the ground state must be delocalised across the double-well potential. As such, we expect the spectrum to follow $C_{2 v}$ selection rules. For $9 \mathrm{H}-\mathrm{An}$, the frequencies were also calculated in the $C_{2 v}$ geometry to obtain their symmetries, and were correlated with the $\mathrm{C}_{s}$ frequencies by visual inspection of the normal modes. In brief, the aforementioned progressions near $19700 \mathrm{~cm}^{-1}$ and $20100 \mathrm{~cm}^{-1}$ for $9 \mathrm{H}-$ An mimic the origin region almost exactly and are readily assigned to the totally symmetric vibrations $\nu_{30}$ and $\nu_{19}$. The third band in the origin progression is not always apparent in the higher vibrations, but it is also much weaker than the first two. That these two progressions should resemble the origin is due to the fact that they are fully symmetric normal modes, having $a_{1}$ symmetry in the $C_{2 v}$ saddle point geometry and $a^{\prime}$ in $\mathrm{C}_{s}$. Another pair of bands at 19300 $\mathrm{cm}^{-1}$ is therefore also readily assigned to $\nu_{37}$ built upon a fully symmetric vibration, $\nu_{35}$.

Several other bands visible for $9 \mathrm{H}-\mathrm{An}$ in Figure 1 do not show this clear correlation with the origin progression and assignments to these are thus less secure. Nonetheless we have found reasonable assignments for these transitions by making the assumption that the only modes that show up as single quanta correlate to $a_{1}$, while $b_{1}$ modes combine with odd quanta of $\nu_{37}$. Note that $b_{1}$ modes also correlate with $a^{\prime}$ in the bent geometry. However, we expect the $C_{2 v}$ selection rules to hold due to the proposed $a_{1}$ symmetry of the ground state. The $\nu_{37}$ potential is likely coupled to other modes, rendering the appearance of its progression sensitive to combination with these modes. Several examples are apparent in the deuterated isotopologue and we will return to this in the Discussion. The mean average deviation (MAD) between theory and calculation is $5.4 \mathrm{~cm}^{-1}$ for the scaled $C_{s}$ frequencies which, considering the size of the molecule and the degree of anharmonicity associated with the floppy molecular frame, is acceptable. 
Table 2: $9 \mathrm{H}-\mathrm{An}$ Spectral Assignments $\left(\mathrm{cm}^{-1}\right)$ and Comparison to Calculations.

\begin{tabular}{rrcrr}
\hline \hline Observed & Relative & Assignment & Calc. $C_{s}$ & $\Delta$ \\
\hline & & & & \\
19114.8 & 0 & $0_{0}^{0}$ & 0 & \\
19141.4 & 26.6 & $37_{0}^{2}$ & $26.6 \dagger$ & \\
19182.6 & 67.7 & $37_{0}^{4}$ & $67.7 \dagger$ & \\
19279.4 & 164.6 & $69_{0}^{2}$ & 162.4 & -2.2 \\
19283.7 & 168.8 & $36_{0}^{1} 37_{0}^{1}$ & 167.8 & -1.0 \\
19296.6 & 181.7 & $69_{0}^{2} 37_{0}^{2 *}$ & 189.0 & \\
19319.1 & 204.2 & $36_{0}^{1} 37_{0}^{3}$ & 203.8 & \\
19349.4 & 234.5 & $35_{0}^{1}$ & 232.1 & -2.4 \\
19377.9 & 263.0 & $35_{0}^{1} 37_{0}^{2}$ & 258.7 & \\
19689.5 & 574.7 & $30_{0}^{1}$ & 565.3 & -9.4 \\
19716 & 601.1 & $30_{0}^{1} 37_{0}^{2}$ & 591.9 & \\
19755.5 & 640.7 & $30_{0}^{1} 37_{0}^{4}$ & 633.0 & \\
19807.7 & 692.8 & $27_{0}^{1} 37_{0}^{1}$ & 692.8 & 0.0 \\
19815.1 & 700.2 & $26_{0}^{1}$ & 692.2 & -8.0 \\
19838.5 & 723.6 & $26_{0}^{1} 37_{0}^{2}$ & 718.8 & \\
19978 & 863.2 & $23_{0}^{1}$ & 869.9 & 6.7 \\
20005.6 & 890.8 & $23_{0}^{1} 37_{0}^{2}$ & 896.5 & \\
20036 & 921.2 & $21_{0}^{1} 37_{0}^{1}$ & 927.6 & 6.4 \\
20096.3 & 981.5 & $19_{0}^{1}$ & 969.2 & -12.3 \\
20122.9 & 1008.1 & $19_{0}^{1} 37_{0}^{2}$ & 995.8 & \\
& $\dagger$ Modelled - see text. & &
\end{tabular}

\section{Discussion}

It is evident from the previous section that deuteration at the 9-position causes a significant change in the effective structure of the radical in its $D_{1}$ state. Knowledge of the ground state is, by the nature of our experiments, limited. But, using the DFT results as a guideline, a similar deuteration effect as seen in $D_{1}$ seems likely. The DFT electronic energies place the 9-carbon distinctly out of plane for both the ground and excited states of the radical, with the excited state more puckered. However, the energy difference between the out-of-plane geometry and the $C_{2 v^{-}}$ planar geometry is very small, and the effective structure of the 9H-An radical is therefore still essentially planar. The vibrational zero-point energy level along the butterfly mode, $\nu_{37}$, lies at 
or just above the barrier to inversion, and the associated wavefunction has equal intensities in both sides of the well.

Replacing one of the 9-carbon hydrogen atoms with a deuterium atom breaks this symmetry. Purely electronically the potential is still totally symmetric, but the vibrational frequencies of the radical are sensitive to whether the D-atom is in the axial position or in the equatorial position and the vibrational zero-point energy, for all modes except the butterfly mode, reflects this difference (see Figure 5). The symmetric double-well potential along the butterfly coordinate has therefore become an asymmetric double well, with a preponderance for the D-atom in the equatorial position, thus changing the effective (vibrationally averaged) geometry of this radical from planar to bent. While within the harmonic approximation the effect of deuteration on the butterfly vibrational motion itself is very small $\left(\sqrt{\mu_{H} / \mu_{D}}=0.96\right)$, the sum of the effect on all 68 other vibrational modes is substantial. Because this is a purely quantum mechanical effect with no classical analogue, it is termed "quantum-induced symmetry breaking".

This term was applied by Schlemmer and co-workers to the effect of deuteration on the IR spectrum of protonated methane $\left(\mathrm{CH}_{5}^{+}\right)$where, for partial deuteration, there was a clear preference for the deuterium to occupy the more tightly bound $\mathrm{CH}_{3}$ moiety within the cation.[25] In their case, the IR spectra for a given partially deuterated isotopologue could be decomposed into spectra due to all the possible isotopomers for that given isotopologue. In other words, despite the zero-point wavefunction of $\mathrm{CH}_{5}^{+}$being spread between all the possible hydrogen exchange symmetries, distinct structures corresponding to D-atoms being localised in a particular location within the molecule were distinguishable.

While the hole-burning experiment confirms that all transitions in the origin progression of 9D-An originate from a single ground state, the excitation spectrum samples both sides of the excited-state potential. Mode 30 is a case in point, where $30_{0}^{1} 37_{0}^{1}$ is observed some $7.5 \mathrm{~cm}^{-1}$ lower in energy than expected based on the origin progression. This is reflected in a substantial 
predicted $\left(12 \mathrm{~cm}^{-1}\right)$ drop in the frequency of $\nu_{30}$ as the $\mathrm{D}$ atom moves from the equatorial to the axial position (Table S1). If the minima are near $q_{37}= \pm 1$, a modelled, this implies a contribution to the linear term in the effective potential from $\nu_{30}$, of $-3 \mathrm{~cm}^{-1} \AA^{-1}(0.5 d \omega / d q)$. But, from Equation 1, it is apparent that occupancy of $v_{30}=1$ will triple the contribution of $\nu_{30}$ to the effective potential for $q_{37}$. As this contributes in the opposite sense to the overall zero-point energy correction, the effect is to flatten the potential and decrease the energy level spacing close to what is observed.

Modes $\nu_{35}$ and $\nu_{19}$ are not calculated to have a significant change in frequency between the axial and equatorial positions. This is reflected in the $\nu_{37}$ progression exhibiting spacings near identical to the origin. Mode $\nu_{23}$ is calculated to gain in frequency along $q_{37}$, yet we assign the spectrum to a spacing pattern similar to $\nu_{30}$. This may point to a misassignment, but we also note that the calculated frequencies are very sensitive to the geometry and (TD)-B3LYP may not capture the full picture.

Finally, we note that several of the ionization efficiency spectra depicted in Figure 3 show structure above the first ionization onset. This is most apparent in the curves using band $d_{d}$ as an intermediate state for 9D-An and the one using band $h_{a}$ as an intermediate for 9H-An. For 9D-An this second onset appears $57 \mathrm{~cm}^{-1}$ above the ionization threshold, while for $9 \mathrm{H}-\mathrm{An}$, the offset is almost double, $110 \mathrm{~cm}^{-1}$. This spacing is again reminiscent of a low-frequency mode being excited in the cation which we surmise to be the equivalent to $\nu_{37}$ of the radical, the butterfly motion. We calculate that the cation ground state is $C_{2 v}$. We postulate a low-frequency butterfly mode of around $60 \mathrm{~cm}^{-1}$, where the $110 \mathrm{~cm}^{-1}$ seen for $9 \mathrm{H}-\mathrm{An}$ once again implies a $\Delta v=2$ selection rule. The fact that this second ionization onset is most prominent for band $\mathrm{d}_{\mathrm{d}}$ in Figure 3 can be rationalized with an appreciable geometry change along the butterfly mode upon ionization, which is also born out by the slow rising slope of the ionization spectrum for the origin band of 9D-An $\left(\mathrm{d}_{\mathrm{a}}\right)$. The electronic excitation spectra of the closed-shell $9 \mathrm{H}-\mathrm{An}^{+}$and 
9D- $\mathrm{An}^{+}$cations will be reported in a future publication.

\section{Conclusions}

We report the excitation spectra and ionization efficiency curves of two isotopologues of the 9-dihydroanthracenyl radical. Both isotopologues were generated in our laboratory through an electric discharge of a seeded molecular beam containing anthracene vapor as well as normal and deuterated water for 9H-An and 9D-An, respectively. There is no evidence in our spectra for multiple isomers of either species, implying the lowest-energy isomer is exclusively formed and no isotope scrambling occurs in the deuterated species. The experimentally obtained value for the ionization energy of 9H-An is 6.3462(10) eV with the ionization energy of the deuterated isotopologue shifted by $+0.001 \mathrm{eV}$.

Both isotopologues show clear evidence of substantial activity in the low-frequency "butterfly" motion. For 9D-An we observe additional bands in the butterfly progression compared to $9 \mathrm{H}-\mathrm{An}$. These bands are attributed to quantum-induced symmetry breaking of the deuterated isotopologue due to the difference in zero-point energy of the radical with the deuterium atom in the equatorial or axial positions.

\section{Acknowledgements}

This work was supported by the Australian Research Council (DP190103151, Centre of Excellence in Exciton Science CE170100026).

\section{References}

[1] Manjavacas, A. et al. Tunable molecular plasmons in polycyclic aromatic hydrocarbons. ACS Nano 7, 3635-3643 (2013). 
[2] Mokkath, J. H. Optical properties of pyridine adsorbed polycyclic aromatic hydrocarbons using quantum chemical calculations. Phys. Chem. Chem. Phys. 21, 448-454 (2019).

[3] Richter, H. \& Howard, J. Formation of polycyclic aromatic hydrocarbons and their growth to soota review of chemical reaction pathways. Prog. Ener. Combust. Sci. 26, 565-608 (2000).

[4] Johansson, K. O., Head-Gordon, M. P., Schrader, P. E., Wilson, K. R. \& Michelsen, H. A. Resonance-stabilized hydrocarbon-radical chain reactions may explain soot inception and growth. Science 361, 997-1000 (2018).

[5] Ravindra, K., Sokhi, R. \& Van Grieken, R. Atmospheric polycyclic aromatic hydrocarbons: Source attribution, emission factors and regulation. Atmos. Env. 42, 2895-2921 (2008).

[6] McGuire, B. A. et al. Detection of the aromatic molecule benzonitrile $\left(c-\mathrm{C}_{6} \mathrm{H}_{5} \mathrm{CN}\right)$ in the interstellar medium. Science 359, 202-205 (2018).

[7] Tielens, A. Interstellar polycyclic aromatic hydrocarbon molecules. Ann. Rev. Astron. Astrophys. 46, 289-337 (2008).

[8] Mennella, V., Hornekær, L., Thrower, J. \& Accolla, M. The Catalytic Role of Coronene for Molecular Hydrogen Formation. Astrophys. J. 745, L2 (2011).

[9] Page, V. L., Snow, T. P. \& Bierbaum, V. M. Molecular Hydrogen Formation Catalyzed by Polycyclic Aromatic Hydrocarbons in the Interstellar Medium. Astrophys. J. 704, 274-280 (2009).

[10] Foley, N. et al. Molecular hydrogen formation on interstellar PAHs through EleyRideal abstraction reactions. Mon. Not. Roy. Astron. Soc. 479, 649-656 (2018). 
[11] Hornekær, L., Baurichter, A., Petrunin, V. V., Field, D. \& Luntz, A. C. Importance of surface morphology in interstellar $\mathrm{H}_{2}$ formation. Science 302, 1943-1946 (2003).

[12] Sandford, S. A., Bernstein, M. P. \& Dworkin, J. P. Assessment of the interstellar processes leading to deuterium enrichment in meteoritic organics. Meteor. Planet. Sci. 36, $1117-$ $1133(2001)$.

[13] Schmidt, T. W. The electronic spectroscopy of resonance-stabilised hydrocarbon radicals. Int. Rev. Phys. Chem. 35, 209-242 (2016).

[14] Miller, J. A. \& Klippenstein, S. J. The Recombination of Propargyl Radicals: Solving the Master Equation. J. Phys. Chem. A 105, 7254-7266 (2001).

[15] Harding, L. B., Klippenstein, S. J. \& Georgievskii, Y. On the Combination Reactions of Hydrogen Atoms with Resonance-Stabilized Hydrocarbon Radicals. J. Phys. Chem. A 111, 3789-3801 (2007).

[16] Mebel, A. M. \& Kaiser, R. I. Formation of resonantly stabilised free radicals via the reactions of atomic carbon, dicarbon, and tricarbon with unsaturated hydrocarbons: theory and crossed molecular beams experiments. Int. Rev. Phys. Chem. 34, 461-514 (2015).

[17] Nakajima, M., Schmidt, T. W., Sumiyoshi, Y. \& Endo, Y. Rotationally-resolved excitation spectrum of the jet-cooled cyclohexadienyl radical. Chem. Phys. Lett. 449, 57-62 (2007).

[18] Wilcox, C. M., Krechkivska, O., Nauta, K., Schmidt, T. W. \& Kable, S. H. Jet-cooled spectroscopy of ortho-hydroxycyclohexadienyl radicals. J. Phys. Chem. A 122, 88868897 (2018).

[19] Lay, T. H., Bozzelli, J. W. \& Seinfeld, J. H. Atmospheric Photochemical Oxidation of 
Benzene: Benzene $+\mathrm{OH}$ and the Benzene-OH Adduct (Hydroxyl-2,4-cyclohexadienyl) + $\mathrm{O}_{2}$. J. Phys. Chem. 100, 6543-6554 (1996).

[20] Sebree, J. A., Kislov, V. V., Mebel, A. M. \& Zwier, T. S. Spectroscopic and Thermochemical Consequences of Site-Specific H-Atom Addition to Naphthalene. J. Phys. Chem. A 114, 6255-6262 (2010).

[21] Krechkivska, O. et al. Triple-Resonance Spectroscopy Reveals the Excitation Spectrum of Very Cold, Isomer-Specific Protonated Naphthalene. J. Phys. Chem. Lett. 4, 3728-3732 (2013).

[22] Krechkivska, O. et al. H and D Attachment to Naphthalene: Spectra and Thermochemistry of Cold Gas-Phase 1- $\mathrm{C}_{10} \mathrm{H}_{9}$ and 1- $\mathrm{C}_{10} \mathrm{H}_{8} \mathrm{D}$ Radicals and Cations. J. Phys. Chem. A 119, 3225-3232 (2015).

[23] Sebree, J. A., Kislov, V. V., Mebel, A. M. \& Zwier, T. S. Isomer specific spectroscopy of $\mathrm{C}_{10} \mathrm{H}_{n}, \mathrm{n}=8$-12: Exploring pathways to naphthalene in Titan's atmosphere. Faraday Disc. 147, 231 (2010).

[24] Garkusha, I., Fulara, J., Nagy, A. \& Maier, J. P. Electronic Absorption Spectra of Protonated Anthracenes and Phenanthrenes, and Their Neutrals in Neon Matrices. Astrophys. J. 728, 131 (2011).

[25] Ivanov, S. D. et al. Quantum-induced symmetry breaking explains infrared spectra of $\mathrm{CH}_{5}^{+}$isotopologues. Nat. Chem. 2, 298-302 (2010).

[26] Reilly, N. J. et al. Spectroscopic identification of the resonance-stabilized cis- and trans1-vinylpropargyl radicals. J. Amer. Chem. Soc. 131, 13423-13429 (2009). 
[27] Chalyavi, N. et al. Excitation and Emission Spectra of Jet-Cooled Naphthylmethyl Radicals. J. Phys. Chem. A 115, 7959-7965 (2011).

[28] O'Connor, G. D. et al. Excitation Spectra of Large Jet-Cooled Polycyclic Aromatic Hydrocarbon Radicals: 9-Anthracenylmethyl $\left(\mathrm{C}_{15} \mathrm{H}_{11}\right)$ and 1-Pyrenylmethyl $\left(\mathrm{C}_{17} \mathrm{H}_{11}\right) . \quad J$. Phys. Chem. A 117, 13899-13907 (2013).

[29] Nakagawa, K. \& Itoh, N. Analysis of guesthost and intra-guest transitions for hydronaphthyl radicals in naphthalene crystals. Chem. Phys. 16, 461-472 (1976).

[30] Chalyavi, N. et al. Spectroscopy and thermochemistry of a jet-cooled open-shell polyene: 1,4-pentadienyl radical 135, 124306 (2011).

[31] Currie, C. L. \& Ramsay, D. A. Electronic absorption spectrum and dissociation energy of the allyl radical. J. Chem. Phys. 45, 488-491 (1966).

[32] Tsuge, M., Hamatani, S., Kawai, A., Tsuji, K. \& Shibuya, K. Jet spectroscopy of arylmethyl radicals in the visible region: assignment of low-frequency vibrational modes in diphenylmethyl and chlorodiphenylmethyl radicals. Phys. Chem. Chem. Phys. 8, 256-263 (2006).

[33] Krechkivska, O. et al. Ionization Energies of Three Resonance-Stabilized Radicals: Cyclohexadienyl $\left(\mathrm{d}_{n}, \mathrm{n}=0,1,6,7\right)$, 1-Phenylpropargyl, and Methylcyclohexadienyl. J. Phys. Chem. A 118, 10252-10258 (2014).

[34] Frisch, M. J. et al. Gaussian 16 Revision A.03 (2016). Gaussian Inc., 340 Quinnipiac Street, Building 40, Wallingford CT 06492.

[35] Troy, T. P. The Spectroscopy of Radical Chromophores. Ph.D. thesis, The University of Sydney (2011). 
[36] Samanta, A., Bhattacharyya, K. \& Chowdhury, M. Interaction of 2-pi electron systems spectroscopy of 9,10-dihydroanthracene. J. Phys. Chem. 91, 4671-4675 (1987).

[37] Chakraborty, T. \& Chowdhury, M. Fluorescence excitation spectrum of jet-cooled dihydroanthracene. Chem. Phys. Lett. 171, 25-28 (1990).

[38] Shin, Y. D., Saigusa, H., Zgierski, M. Z., Zerbetto, F. \& Lim, E. C. Inversion potentials in the ground and excited states of 9,10-dihydroanthracene as probed by the absorption and excitation spectra of jet-cooled molecules. J. Chem. Phys. (199).

[39] Laane, J. Vibrational Potential Energy Surfaces and Conformations of Molecules in Ground and Excited Electronic States. Ann. Rev. Phys. Chem. 45, 179-211 (1994).

[40] Verguilla-Berdecia, L. A. Tunneling in a quartic, symmetric, double well potential: A simple solution using a hermite basis. J. Chem. Educ. 70, 928 (1993). 


\section{Supporting information for:}

\section{Quantum-induced symmetry breaking in the deuterated dihydroanthracenyl radical}

Olha Krechkivska, ${ }^{\dagger}$ Callan M. Wilcox,${ }^{\dagger}$ Klaas Nauta,${ }^{\dagger}$ Scott H. Kable,${ }^{\dagger}$ and Timothy W. Schmidt*,

†School of Chemistry, UNSW Sydney, NSW 2052, Australia

$\ddagger A R C$ Centre of Excellence in Exciton Science, School of Chemistry, UNSW Sydney, NSW 2052, Australia

E-mail: timothy.schmidt@unsw.edu.au

Phone: +61 439386109 


\section{Detailed Assignments}

The read is referred to Figure S1. As stated in the main text, the lowest calculated frequency is $\nu_{37}$, which is $b_{1}$ in $C_{2 v}$ and $a^{\prime}$ in $C_{s}$. As such, in the absence of multiple isomers, it must be responsible for the short progression between 19100 and $19200 \mathrm{~cm}^{-1}$. As discussed, the holeburning experiments and the band-doubling in 9D-An lead us to assign the progression to even quanta in this mode, implying an $a_{1}$ ground state vibrational wavefunction in $C_{2 v}$. We therefore expect selection rules in this point group to apply. This mode is clearly anharmonic, which is a common characteristic of low-frequency out-plane modes of PAHs.

The spectrum is expected to be dominated by upper vibrational states of $a_{1}$ symmetry. The frequencies may be sensitive to motion along the $q_{37}$ coordinate so we inform our assignments using both $C_{2 v}$ and $C_{s}$ optimized frequencies. Several bands in 9H-An are readily assigned to $a_{1}$ modes 35, 30, 26, 23 and 19, also exhibiting the progression in $\nu_{37}$.

The lowest frequency mode of $a_{1}$ symmetry is $\nu_{35}$, and therefore all band lower in frequency but be built on $a_{1}$ combinations (discounting Herzberg-Teller coupling). Mode 36 is a $b_{1}$ mode which may be combined with odd quanta of $\nu_{37}$. The only other $a_{1}$ combination at these low frequencies is $2 \nu_{69}$, onto which even quanta of $\nu_{37}$ may be built. The cluster of bands around $+200 \mathrm{~cm}^{-1}$ is thus attributed to progression in $\nu_{37}$, built onto $2 \nu_{69}$ (even quanta) and $\nu_{36}$ (odd quanta). The remaining intense features in the $9 \mathrm{H}-\mathrm{An}$ spectrum are assigned to single quanta in $b_{1}$ modes 27 and 21 , coupled to $\nu_{37}$.

The spectrum of 9D-An was assigned with reference to 9H-An. The latter, the assignment of $30_{0}^{1} 37_{0}^{2 n}$ was unambiguous. This region of the 9D-An spectrum reveals at least one more band, as expected due to the quantum-induced symmetry breaking. But, the spacings are not the same as the origin region, serving as a warning that the $\nu_{37}$ potential is sensitive to anharmonic couplings. With this in mind, the remainder of the spectrum was assigned. 


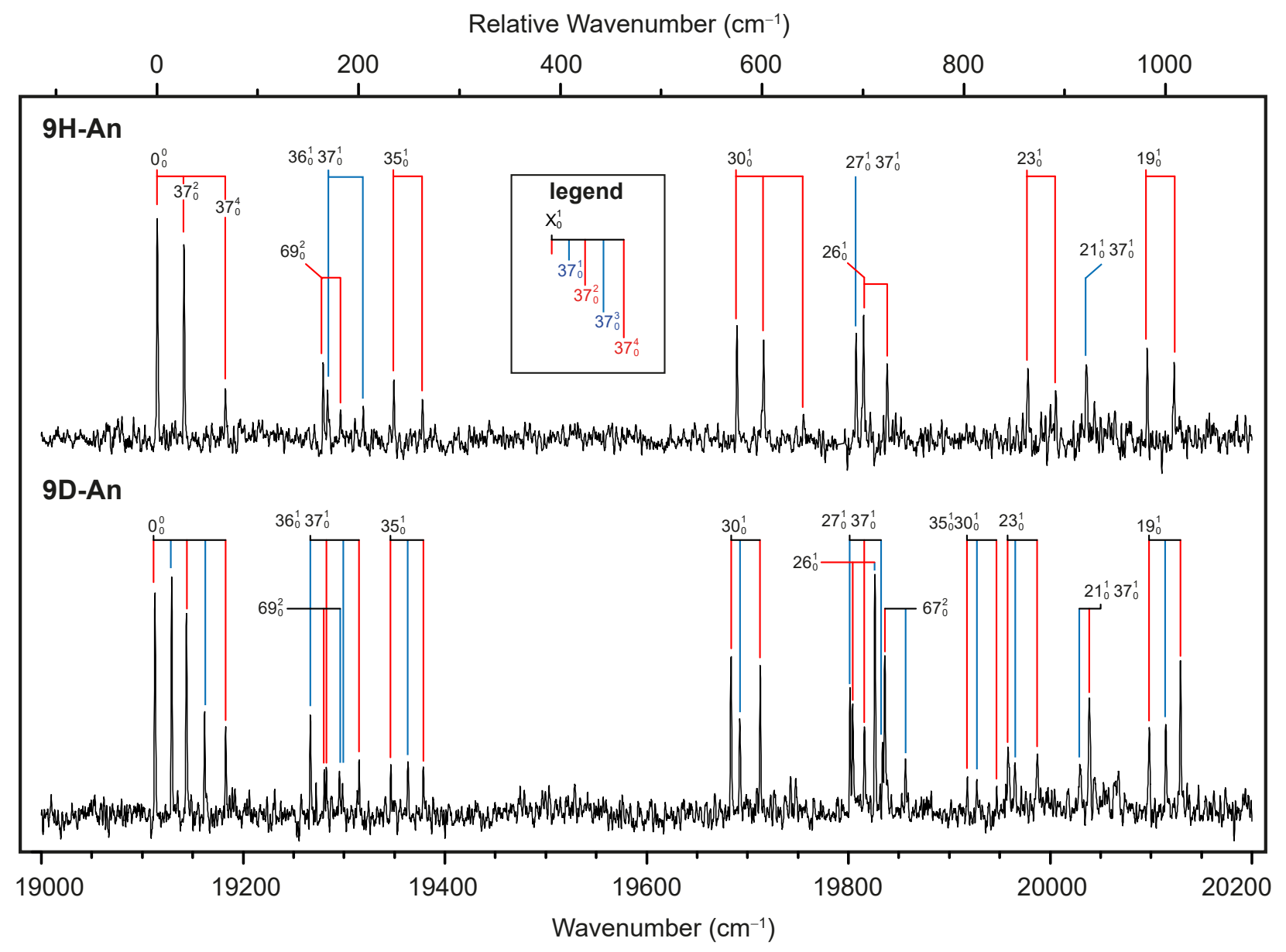

Figure S1: R2C2PI spectra and assignments of the 9H-An and 9D-An radicals. 
Table S1: 9D-An Spectral Assignments $\left(\mathrm{cm}^{-1}\right)$ and Comparison to Calculations.

\begin{tabular}{rrrrrrr}
\hline \hline Observed & Relative & Assignment & Equat. Calc. & $\Delta$ & Axial Calc & $\Delta$ \\
\hline 19112.5 & 0 & $0_{0}^{0}$ & 0 & & & \\
19129.2 & 16.6 & $37_{0}^{1}$ & 16.4 & -0.2 & & \\
19143.8 & 31.3 & $37_{0}^{2}$ & 31.2 & -0.1 & & \\
19161.7 & 49.2 & $37_{0}^{3}$ & 49.6 & 0.3 & & \\
19182.8 & 70.3 & $37_{0}^{4}$ & 70.3 & 0.0 & & \\
19266.8 & 154.2 & $36_{0}^{1}$ & 151.5 & -2.7 & 150.4 & -3.8 \\
19282.5 & 170.0 & $36_{0}^{1} 37_{0}^{1}$ & 167.9 & -2.1 & 166.8 & -3.2 \\
19298.5 & 185.9 & $36_{0}^{1} 37_{0}^{2}$ & 182.7 & -3.2 & 181.6 & -4.3 \\
19314.9 & 202.4 & $36_{0}^{1} 37_{0}^{3}$ & 201.1 & -1.3 & 200.0 & -2.4 \\
19280.5 & 168.0 & $69_{0}^{2}$ & 162.6 & -5.4 & 162.4 & -5.6 \\
19295.6 & 183.0 & $69_{0}^{2} 37_{0}^{1}$ & 179.0 & -4.0 & 178.8 & -4.2 \\
19346.4 & 233.9 & $35_{0}^{1}$ & 228.9 & -5.0 & 231.2 & -2.7 \\
19363.3 & 250.7 & $350_{0}^{1} 37_{0}^{1}$ & 245.3 & -5.4 & 247.6 & -3.1 \\
19378.7 & 266.1 & $35_{0}^{1} 37_{0}^{2}$ & 260.1 & -6.0 & 262.4 & -3.7 \\
19684.0 & 571.5 & $30_{0}^{1}$ & 563.1 & -8.4 & 551.1 & -20.4 \\
19692.9 & 580.4 & $30_{0}^{1} 37_{0}^{1}$ & 579.5 & -0.9 & 567.5 & -12.9 \\
19712.9 & 600.4 & $30_{0}^{1} 37_{0}^{2}$ & 594.3 & -6.1 & 582.3 & -18.1 \\
19801.3 & 688.8 & $27_{0}^{1} 37_{0}^{1}$ & 695.3 & 6.5 & 696.3 & 7.5 \\
19815.7 & 703.1 & $27_{0}^{1} 37_{0}^{2}$ & 710.1 & 7.0 & 711.1 & 8.0 \\
19833.9 & 721.4 & $27_{0}^{1} 37_{0}^{3}$ & 728.5 & 7.1 & 729.5 & 8.1 \\
19804.4 & 691.8 & $26_{0}^{1}$ & 686.4 & -5.4 & 691.9 & 0.1 \\
19826.6 & 714.1 & $26_{0}^{1} 37_{0}^{1}$ & 702.8 & -11.3 & 708.3 & -5.8 \\
19836.3 & 723.7 & $67_{0}^{2}$ & 724.6 & 0.9 & 728.6 & 4.9 \\
19856.6 & 744.0 & $67_{0}^{2} 37_{0}^{1}$ & 741.0 & -3.0 & 745.0 & 1.0 \\
19918.0 & 805.4 & $30_{0}^{1} 35_{0}^{1}$ & 792.0 & -13.4 & 782.3 & -23.1 \\
19927.6 & 815.1 & $30_{0}^{1} 35_{0}^{1} 37_{0}^{1}$ & 808.4 & -6.7 & 798.7 & -16.4 \\
19947.0 & 834.4 & $30_{0}^{1} 35_{0}^{1} 37_{0}^{2}$ & 839.6 & 5.2 & 829.9 & -4.5 \\
19958.4 & 845.8 & $23_{0}^{1}$ & 857.4 & 11.6 & 866.9 & 21.1 \\
19965.3 & 852.8 & $23_{0}^{1} 37_{0}^{1}$ & 873.8 & 21.0 & 883.3 & 30.5 \\
19987.4 & 874.8 & $23_{0}^{1} 37_{0}^{2}$ & 888.6 & 13.8 & 898.1 & 23.3 \\
20029.7 & 917.1 & $21_{0}^{1} 37_{0}^{1}$ & 921.6 & 4.5 & 919.6 & 2.5 \\
20038.8 & 926.2 & $21_{0}^{1} 37_{0}^{2}$ & 936.4 & 10.2 & 934.4 & 8.2 \\
20098.2 & 985.6 & $19_{0}^{1}$ & 969.0 & -16.6 & 969.1 & -16.5 \\
20114.6 & 1002.0 & $19_{0}^{1} 37_{0}^{1}$ & 985.4 & -16.6 & 985.5 & -16.5 \\
20129.1 & 1016.6 & $19_{0}^{1} 37_{0}^{2}$ & 1000.2 & -16.4 & 1000.3 & -16.3
\end{tabular}




\section{Calculated Frequencies}

Table S2: TD-B3LYP/6-311+G(d,p) Vibrational frequencies for the $\mathrm{D}_{1}$ electronic state of the $9 \mathrm{H}-$ An radical

\begin{tabular}{ccccccccc}
\hline \hline Mode \# & Frequency & Symmetry & Mode \# & Frequency & Symmetry & Mode \# & Frequency & Symmetry \\
\hline 69 & 83.7 & $\mathrm{a}^{\prime \prime}$ & 46 & 1383.4 & $\mathrm{a}^{\prime \prime}$ & 23 & 896.8 & $\mathrm{a}^{\prime}$ \\
68 & 219.3 & $\mathrm{a}^{\prime \prime}$ & 45 & 1436.5 & $\mathrm{a}^{\prime \prime}$ & 22 & 900.4 & $\mathrm{a}^{\prime}$ \\
67 & 375.5 & $\mathrm{a}^{\prime \prime}$ & 44 & 1499.9 & $\mathrm{a}^{\prime \prime}$ & 21 & 946.2 & $\mathrm{a}^{\prime}$ \\
66 & 391.0 & $\mathrm{a}^{\prime \prime}$ & 43 & 1543.8 & $\mathrm{a}^{\prime \prime}$ & 20 & 962.5 & $\mathrm{a}^{\prime}$ \\
65 & 444.8 & $\mathrm{a}^{\prime \prime}$ & 42 & 1663.4 & $\mathrm{a}^{\prime \prime}$ & 19 & 999.2 & $\mathrm{a}^{\prime}$ \\
64 & 504.8 & $\mathrm{a}^{\prime \prime}$ & 41 & 3148.5 & $\mathrm{a}^{\prime \prime}$ & 18 & 1067.2 & $\mathrm{a}^{\prime}$ \\
63 & 567.5 & $\mathrm{a}^{\prime \prime}$ & 40 & 3157.7 & $\mathrm{a}^{\prime \prime}$ & 17 & 1163.2 & $\mathrm{a}^{\prime}$ \\
62 & 654.5 & $\mathrm{a}^{\prime \prime}$ & 39 & 3179.3 & $\mathrm{a}^{\prime \prime}$ & 16 & 1186.1 & $\mathrm{a}^{\prime}$ \\
61 & 710.9 & $\mathrm{a}^{\prime \prime}$ & 38 & 3194.2 & $\mathrm{a}^{\prime \prime}$ & 15 & 1273.0 & $\mathrm{a}^{\prime}$ \\
60 & 719.9 & $\mathrm{a}^{\prime \prime}$ & 37 & 37.1 & $\mathrm{a}^{\prime}$ & 14 & 1312.5 & $\mathrm{a}^{\prime}$ \\
59 & 800.9 & $\mathrm{a}^{\prime \prime}$ & 36 & 162.9 & $\mathrm{a}^{\prime}$ & 13 & 1363.5 & $\mathrm{a}^{\prime}$ \\
58 & 817.1 & $\mathrm{a}^{\prime \prime}$ & 35 & 239.3 & $\mathrm{a}^{\prime}$ & 12 & 1389.8 & $\mathrm{a}^{\prime}$ \\
57 & 903.0 & $\mathrm{a}^{\prime \prime}$ & 34 & 278.2 & $\mathrm{a}^{\prime}$ & 11 & 1441.5 & $\mathrm{a}^{\prime}$ \\
56 & 920.8 & $\mathrm{a}^{\prime \prime}$ & 33 & 372.3 & $\mathrm{a}^{\prime}$ & 10 & 1469.0 & $\mathrm{a}^{\prime}$ \\
55 & 958.5 & $\mathrm{a}^{\prime \prime}$ & 32 & 405.5 & $\mathrm{a}^{\prime}$ & 9 & 1516.9 & $\mathrm{a}^{\prime}$ \\
54 & 964.4 & $\mathrm{a}^{\prime \prime}$ & 31 & 479.9 & $\mathrm{a}^{\prime}$ & 8 & 1616.5 & $\mathrm{a}^{\prime}$ \\
53 & 1101.0 & $\mathrm{a}^{\prime \prime}$ & 30 & 582.8 & $\mathrm{a}^{\prime}$ & 7 & 2950.5 & $\mathrm{a}^{\prime}$ \\
52 & 1178.9 & $\mathrm{a}^{\prime \prime}$ & 29 & 635.4 & $\mathrm{a}^{\prime}$ & 6 & 3035.7 & $\mathrm{a}^{\prime}$ \\
51 & 1192.7 & $\mathrm{a}^{\prime \prime}$ & 28 & 650.2 & $\mathrm{a}^{\prime}$ & 5 & 3149.0 & $\mathrm{a}^{\prime}$ \\
50 & 1224.0 & $\mathrm{a}^{\prime \prime}$ & 27 & 704.2 & $\mathrm{a}^{\prime}$ & 4 & 3149.2 & $\mathrm{a}^{\prime}$ \\
49 & 1243.4 & $\mathrm{a}^{\prime \prime}$ & 26 & 713.6 & $\mathrm{a}^{\prime}$ & 3 & 3158.6 & $\mathrm{a}^{\prime}$ \\
48 & 1297.2 & $\mathrm{a}^{\prime \prime}$ & 25 & 789.5 & $\mathrm{a}^{\prime}$ & 2 & 3179.6 & $\mathrm{a}^{\prime}$ \\
47 & 1355.9 & $\mathrm{a}^{\prime \prime}$ & 24 & 866.1 & $\mathrm{a}^{\prime}$ & 1 & 3195.3 & $\mathrm{a}^{\prime}$
\end{tabular}


Table S3: TD-B3LYP/6-311+G(d,p) Vibrational frequencies for the $\mathrm{D}_{1}$ electronic state of the $9 \mathrm{D}_{e q^{-}}$ An radical

\begin{tabular}{ccccccccc}
\hline \hline Mode \# & Frequency & Symmetry & Mode \# & Frequency & Symmetry & Mode \# & Frequency & Symmetry \\
\hline 69 & 83.8 & $\mathrm{a}^{\prime \prime}$ & 46 & 1374.2 & $\mathrm{a}^{\prime \prime}$ & 23 & 883.9 & $\mathrm{a}^{\prime}$ \\
68 & 219.6 & $\mathrm{a}^{\prime \prime}$ & 45 & 1430.4 & $\mathrm{a}^{\prime \prime}$ & 22 & 898.8 & $\mathrm{a}^{\prime}$ \\
67 & 373.5 & $\mathrm{a}^{\prime \prime}$ & 44 & 1497.6 & $\mathrm{a}^{\prime \prime}$ & 21 & 933.2 & $\mathrm{a}^{\prime}$ \\
66 & 391.3 & $\mathrm{a}^{\prime \prime}$ & 43 & 1543.7 & $\mathrm{a}^{\prime \prime}$ & 20 & 962.2 & $\mathrm{a}^{\prime}$ \\
65 & 443.7 & $\mathrm{a}^{\prime \prime}$ & 42 & 1663.1 & $\mathrm{a}^{\prime \prime}$ & 19 & 998.9 & $\mathrm{a}^{\prime}$ \\
64 & 497.2 & $\mathrm{a}^{\prime \prime}$ & 41 & 3148.5 & $\mathrm{a}^{\prime \prime}$ & 18 & 1067.2 & $\mathrm{a}^{\prime}$ \\
63 & 567.2 & $\mathrm{a}^{\prime \prime}$ & 40 & 3157.7 & $\mathrm{a}^{\prime \prime}$ & 17 & 1158.4 & $\mathrm{a}^{\prime}$ \\
62 & 648.1 & $\mathrm{a}^{\prime \prime}$ & 39 & 3179.3 & $\mathrm{a}^{\prime \prime}$ & 16 & 1183.3 & $\mathrm{a}^{\prime}$ \\
61 & 699.9 & $\mathrm{a}^{\prime \prime}$ & 38 & 3194.2 & $\mathrm{a}^{\prime \prime}$ & 15 & 1272.0 & $\mathrm{a}^{\prime}$ \\
60 & 711.9 & $\mathrm{a}^{\prime \prime}$ & 37 & 35.9 & $\mathrm{a}^{\prime}$ & 14 & 1312.7 & $\mathrm{a}^{\prime}$ \\
59 & 800.9 & $\mathrm{a}^{\prime \prime}$ & 36 & 156.2 & $\mathrm{a}^{\prime}$ & 13 & 1364.3 & $\mathrm{a}^{\prime}$ \\
58 & 808.0 & $\mathrm{a}^{\prime \prime}$ & 35 & 235.9 & $\mathrm{a}^{\prime}$ & 12 & 1390.1 & $\mathrm{a}^{\prime}$ \\
57 & 884.1 & $\mathrm{a}^{\prime \prime}$ & 34 & 273.1 & $\mathrm{a}^{\prime}$ & 11 & 1281.4 & $\mathrm{a}^{\prime}$ \\
56 & 917.8 & $\mathrm{a}^{\prime \prime}$ & 33 & 371.7 & $\mathrm{a}^{\prime}$ & 10 & 1466.4 & $\mathrm{a}^{\prime}$ \\
55 & 958.6 & $\mathrm{a}^{\prime \prime}$ & 32 & 404.1 & $\mathrm{a}^{\prime}$ & 9 & 1516.9 & $\mathrm{a}^{\prime}$ \\
54 & 949.6 & $\mathrm{a}^{\prime \prime}$ & 31 & 477.7 & $\mathrm{a}^{\prime}$ & 8 & 1615.9 & $\mathrm{a}^{\prime}$ \\
53 & 1101.0 & $\mathrm{a}^{\prime \prime}$ & 30 & 580.5 & $\mathrm{a}^{\prime}$ & 7 & 2951.3 & $\mathrm{a}^{\prime}$ \\
52 & 1179.4 & $\mathrm{a}^{\prime \prime}$ & 29 & 638.1 & $\mathrm{a}^{\prime}$ & 6 & 2229.0 & $\mathrm{a}^{\prime}$ \\
51 & 985.8 & $\mathrm{a}^{\prime \prime}$ & 28 & 628.8 & $\mathrm{a}^{\prime}$ & 5 & 3148.9 & $\mathrm{a}^{\prime}$ \\
50 & 1216.5 & $\mathrm{a}^{\prime \prime}$ & 27 & 699.9 & $\mathrm{a}^{\prime}$ & 4 & 3149.2 & $\mathrm{a}^{\prime}$ \\
49 & 1242.9 & $\mathrm{a}^{\prime \prime}$ & 26 & 707.7 & $\mathrm{a}^{\prime}$ & 3 & 3158.6 & $\mathrm{a}^{\prime}$ \\
48 & 1289.3 & $\mathrm{a}^{\prime \prime}$ & 25 & 785.9 & $\mathrm{a}^{\prime}$ & 2 & 3179.6 & $\mathrm{a}^{\prime}$ \\
47 & 1343.2 & $\mathrm{a}^{\prime \prime}$ & 24 & 832.4 & $\mathrm{a}^{\prime}$ & 1 & 3195.3 & $\mathrm{a}^{\prime}$
\end{tabular}


Table S4: TD-B3LYP/6-311+G(d,p) Vibrational frequencies for the $\mathrm{D}_{1}$ electronic state of the $9 \mathrm{D}_{a x}$-An radical

\begin{tabular}{ccccccccc}
\hline \hline Mode \# & Frequency & Symmetry & Mode \# & Frequency & Symmetry & Mode \# & Frequency & Symmetry \\
\hline 69 & 83.7 & $\mathrm{a}^{\prime \prime}$ & 46 & 1380.8 & $\mathrm{a}^{\prime \prime}$ & 23 & 893.8 & $\mathrm{a}^{\prime}$ \\
68 & 218.6 & $\mathrm{a}^{\prime \prime}$ & 45 & 1435.3 & $\mathrm{a}^{\prime \prime}$ & 22 & 899.0 & $\mathrm{a}^{\prime}$ \\
67 & 375.6 & $\mathrm{a}^{\prime \prime}$ & 44 & 1500.1 & $\mathrm{a}^{\prime \prime}$ & 21 & 931.1 & $\mathrm{a}^{\prime}$ \\
66 & 391.3 & $\mathrm{a}^{\prime \prime}$ & 43 & 1543.6 & $\mathrm{a}^{\prime \prime}$ & 20 & 962.2 & $\mathrm{a}^{\prime}$ \\
65 & 439.1 & $\mathrm{a}^{\prime \prime}$ & 42 & 1663.4 & $\mathrm{a}^{\prime \prime}$ & 19 & 999.1 & $\mathrm{a}^{\prime}$ \\
64 & 501.2 & $\mathrm{a}^{\prime \prime}$ & 41 & 3148.5 & $\mathrm{a}^{\prime \prime}$ & 18 & 1066.9 & $\mathrm{a}^{\prime}$ \\
63 & 566.8 & $\mathrm{a}^{\prime \prime}$ & 40 & 3157.8 & $\mathrm{a}^{\prime \prime}$ & 17 & 1162.6 & $\mathrm{a}^{\prime}$ \\
62 & 647.6 & $\mathrm{a}^{\prime \prime}$ & 39 & 3179.3 & $\mathrm{a}^{\prime \prime}$ & 16 & 1185.6 & $\mathrm{a}^{\prime}$ \\
61 & 683.7 & $\mathrm{a}^{\prime \prime}$ & 38 & 3194.2 & $\mathrm{a}^{\prime \prime}$ & 15 & 1272.9 & $\mathrm{a}^{\prime}$ \\
60 & 713.5 & $\mathrm{a}^{\prime \prime}$ & 37 & 36.0 & $\mathrm{a}^{\prime}$ & 14 & 1312.5 & $\mathrm{a}^{\prime}$ \\
59 & 800.0 & $\mathrm{a}^{\prime \prime}$ & 36 & 155.0 & $\mathrm{a}^{\prime}$ & 13 & 1364.4 & $\mathrm{a}^{\prime}$ \\
58 & 816.8 & $\mathrm{a}^{\prime \prime}$ & 35 & 238.4 & $\mathrm{a}^{\prime}$ & 12 & 1391.7 & $\mathrm{a}^{\prime}$ \\
57 & 888.2 & $\mathrm{a}^{\prime \prime}$ & 34 & 271.3 & $\mathrm{a}^{\prime}$ & 11 & 1279.3 & $\mathrm{a}^{\prime}$ \\
56 & 914.6 & $\mathrm{a}^{\prime \prime}$ & 33 & 370.4 & $\mathrm{a}^{\prime}$ & 10 & 1467.1 & $\mathrm{a}^{\prime}$ \\
55 & 960.2 & $\mathrm{a}^{\prime \prime}$ & 32 & 402.6 & $\mathrm{a}^{\prime}$ & 9 & 1516.9 & $\mathrm{a}^{\prime}$ \\
54 & 954.5 & $\mathrm{a}^{\prime \prime}$ & 31 & 475.6 & $\mathrm{a}^{\prime}$ & 8 & 1616.4 & $\mathrm{a}^{\prime}$ \\
53 & 1102.0 & $\mathrm{a}^{\prime \prime}$ & 30 & 568.2 & $\mathrm{a}^{\prime}$ & 7 & 2163.0 & $\mathrm{a}^{\prime}$ \\
52 & 1182.3 & $\mathrm{a}^{\prime \prime}$ & 29 & 643.2 & $\mathrm{a}^{\prime}$ & 6 & 3034.7 & $\mathrm{a}^{\prime}$ \\
51 & 973.0 & $\mathrm{a}^{\prime \prime}$ & 28 & 631.8 & $\mathrm{a}^{\prime}$ & 5 & 3149.0 & $\mathrm{a}^{\prime}$ \\
50 & 1208.5 & $\mathrm{a}^{\prime \prime}$ & 27 & 700.9 & $\mathrm{a}^{\prime}$ & 4 & 3149.2 & $\mathrm{a}^{\prime}$ \\
49 & 1242.6 & $\mathrm{a}^{\prime \prime}$ & 26 & 713.3 & $\mathrm{a}^{\prime}$ & 3 & 3158.6 & $\mathrm{a}^{\prime}$ \\
48 & 1297.4 & $\mathrm{a}^{\prime \prime}$ & 25 & 786.6 & $\mathrm{a}^{\prime}$ & 2 & 3179.6 & $\mathrm{a}^{\prime}$ \\
47 & 1352.6 & $\mathrm{a}^{\prime \prime}$ & 24 & 829.2 & $\mathrm{a}^{\prime}$ & 1 & 3195.3 & $\mathrm{a}^{\prime}$
\end{tabular}


H+Anthracene Radical

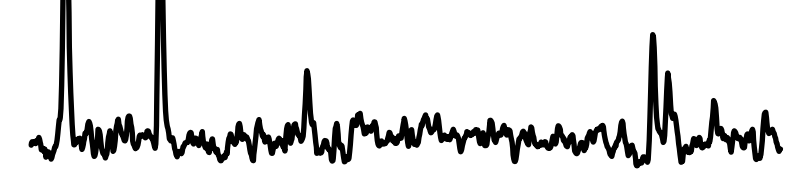
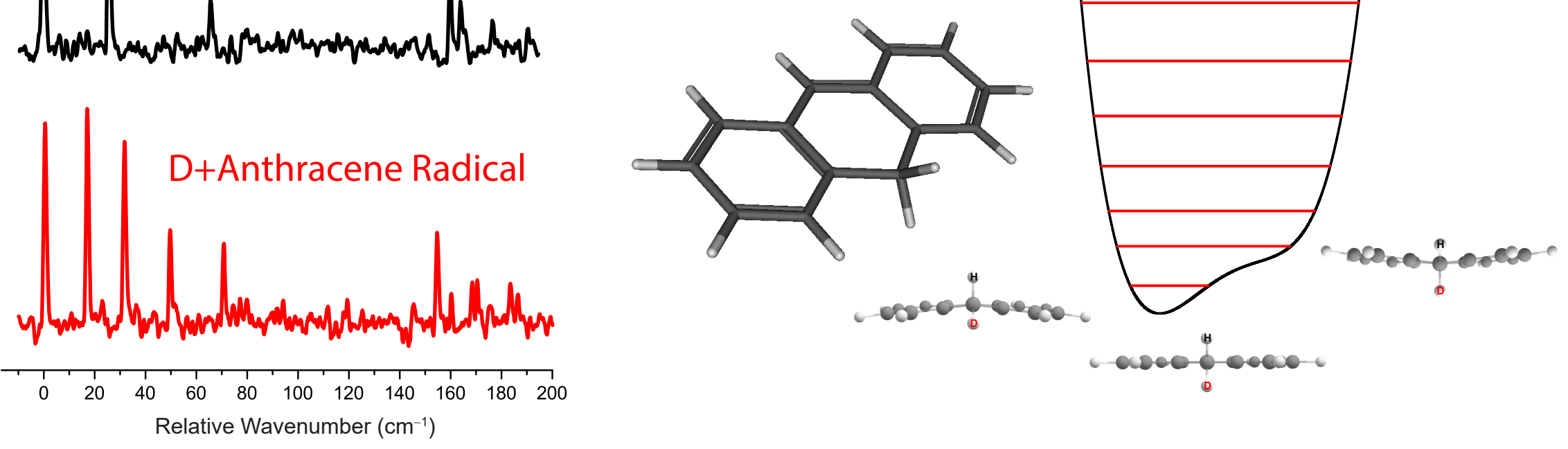
